SUPPORTING INFORMATION

\title{
PERFLUOROALKYL CONTAMINANTS IN THE CANADIAN ARCTIC: EVIDENCE OF ATMOSPHERIC TRANSPORT AND LOCAL CONTAMINATION
}

\author{
Naomi L. Stock ${ }^{\dagger}$, Vasile I. Furdui ${ }^{\dagger}$, Derek C. G. Muir ${ }^{\circ}$ and Scott A. Mabury ${ }^{\dagger} *$ \\ ${ }^{\dagger}$ Department of Chemistry, University of Toronto, 80 St George Street, Toronto Ontario Canada \\ M5S 3H6, and \\ ○ Water Science and Technology Directorate, Environment Canada, 867 Lakeshore Drive, \\ Burlington Ontario, Canada L7R 4A6 \\ * author to whom correspondence should be addressed \\ Phone: (416) 978-3566 Fax: (416) 978-3596 e-mail: smabury@chem.utoronto.ca
}

\section{Contents:}

Chemicals and reagents.

Sediment dating.

Sample collection and preparation.

Extraction, analysis and quality control.

Table S1. Characteristics of Amituk, Char and Resolute Lakes.

Table S2. LODs, LOQs and extraction recoveries for water, sediment and air samples.

Table S3. Concentrations of PFSAs and PFCAs in water samples.

Table S4. Concentrations of PFSAS and PFCAS in sediment samples.

Table S5. Concentrations of individual FTOHs and FSAs in air samples.

Figure S1. Back trajectory probability map of air sampling location.

Table S6. Concentrations of chlorophyll A, chloride and sodium in various Arctic Lakes. 


\section{Chemicals and Reagents}

Optima grade methyl tert-butyl ether, methanol and ethyl acetate (all $99.9 \%$ ) were obtained from Fisher Scientific (Toronto, ON). Perfluoroheptanoic acid (PFHpA, 99 \%), perfluorooctanoic acid (PFOA, 96 \%), perfluorononanoic (PFNA, 97 \%), perfluorodecanoic acid (PFDA, 98 \%), perfluoroundecanoic acid (PFUnA, 95 \%), perfluorododecanoic acid (PFDoA, 95 \%), perfluorotetradecanoic acid (PFTeA 97 \%), potassium PFDS (25 \%), nonadecafluoro-1decanol (NDFD) (98\%), sodium carbonate and tetrabutylammonium hydrogen sulfate (TBAS) were purchased from Sigma-Aldrich (Oakville, ON). Perfluorohexane sulfonate (PFHxS, 99.9 \%), potassium perfluorooctane sulfonate (PFOS, $86.4 \%$ ), perfluorooctane sulfonamide (PFOSA, $>90 \%$ ), $N$-methyl perfluorobutanesulfonamidoethanol (NMeFBSE, $95.55 \%$ ), $N$-methyl perfluorooctanesulfonamidoethanol (NMeFOSE, $96.32 \%$ ) and $N$-ethyl perfluorooctanesulfonamidoethanol (NEtFOSE, $97 \%$ ) standards were donated by 3M Company (St. Paul, MN). Unsaturated fluorotelomer acids (6:2 8:2 and 10:2 FTUCAs) and isotopicallylabeled $\left({ }^{13} \mathrm{C}\right)$ standards $\left({ }^{13} \mathrm{C}_{4}\right.$-PFOA, ${ }^{13} \mathrm{C}_{5}$-PFNA and ${ }^{13} \mathrm{C}_{2}$-PFDA, ${ }^{13} \mathrm{C}_{2}-6: 2$ FTUCA, ${ }^{13} \mathrm{C}_{2}-8: 2$ FTUCA and ${ }^{13} \mathrm{C}_{2}$-10:2 FTUCA, ${ }^{13} \mathrm{C}_{4}$-PFOS) were donated by Wellington Labs (Guelph, ON). Fluorotelomer alcohol standards (6:2, 8:2 and 10:2 FTOHs, all 97\%) were purchased from Oakwood Research Chemicals (West Columbia, SC). $N$-ethyl perfluorooctanesulfonamide (NEtFOSA, $95 \%$ ) were purchased from Lancaster Synthesis (Pelham, NH). N-ethyl perfluorobutanesulfonamidoethanol (NEtFBSE) was not commercially available and was synthesized in our lab. High purity nitrogen was obtained from BOC gases (Whitby, ON). All chemicals were used as received. 


\section{Sample Collection and Preparation}

An inflatable boat was used to collect water and sediment samples from all lakes.

Triplicate water samples were collected approximately $0.5 \mathrm{~m}$ below surface in methanol-rinsed polyethylene bottles. Duplicate sediment cores $(7 \mathrm{~cm}$ diameter) were collected at the deepest point in each of Amituk and Char Lakes using a KB corer, in methanol-rinsed polycarbonate tubes. Cores were hydraulically extruded for sectioning. One core from each lake was sectioned at $0.5 \pm 0.1 \mathrm{~cm}$ intervals and used for sediment dating. The other core was sectioned into three 1.0 or $1.5 \mathrm{~cm}$ slices, a middle slice (approximately $10 \mathrm{~cm}$ ) and a bottom slice (approximately 5 $\mathrm{cm}$ ) and used for contaminant analysis. Coarser resolution was used for the cores for contaminant analysis to ensure that concentrations could be measured. Only one core was collected from Resolute Lake and prepared as above for contaminant analysis.

The location used for air sampling has previously been used for the collection of air samples $(1,2)(1,2)$. Ten air samples (each approximately 72 hours) were collected between July 4 to August 11 2004. Sampling rates ranged from 150 to $200 \mathrm{~L} / \mathrm{min}$ and the average sample volume was $675 \mathrm{~m}^{3}$. Air sampling media consisted of a $10 \mathrm{~cm}$ quartz fiber filter (Tisch Environmental) and $25 \mathrm{~g}$ of XAD-2 resin (Supelco, Oakville ON) sandwiched between two 2.5 cm polyurethane foam (PUF) slices (Tisch Environmental). All air sampling media was cleaned prior to use as previously described (3). During and after transfer to the laboratory, samples were stored at $4^{\circ} \mathrm{C}$ prior to extraction and analysis. 


\section{Sediment Dating}

One sediment core from each of Amituk and Char Lakes was dated using the ${ }^{210} \mathrm{~Pb}$ dating technique (3). Excess ${ }^{210} \mathrm{~Pb}$ in each slice was calculated by subtracting the total ${ }^{210} \mathrm{~Pb}$ activity from the activity of measured ${ }^{226} \mathrm{Ra}$. The measured distribution of excess ${ }^{210} \mathrm{~Pb}$ with respect to depth, was fitted with the Constant Rate of Supply (CRS) model (4) to determine sedimentation rates for each lake and median ages for each core slice. The CRS model accounts for sediment compaction and resuspension by dealing with cumulative dry weight instead of sediment depth. The standard error on dates obtained using this model was 1.229. Dates for sediment from Resolute Lake were estimated from a previously dated core collected from the same location (5).

It should be noted that, measured sediment organic carbon normalized distribution coefficients ( $\log \mathrm{K}_{\mathrm{OC}}$ ) of PFSAs and PFCAs range from 2.06 to $3.53 \mathrm{~L} / \mathrm{kg}$ (6) and as such, it is possible that migration of these contaminants within the sediments via diffusion of dissolved and colloid-bound species in porewater may occur (7). 


\section{Extraction, Analysis and Quality Control}

Samples were extracted and analyzed using previously described methods (7-9). No extraction was necessary for water samples, methanol diluted aliquots were injected directly into the instrument. Sediment samples (approximately $7 \mathrm{~g}$ wet weight) were extracted with methyl tert-butyl ether (MTBE) and the ion-pairing agent tetrabutylammonium hydrogen sulfate. Following extraction, the MTBE aliquots were combined, brought to dryness under a gentle stream of high purity nitrogen and reconstituted in $0.5 \mathrm{~mL}$ methanol. Air samples were extracted in a laboratory clean room using methanol and ethyl acetate. The particulate (quartz fiber filter) and gas (PUF-XAD sandwich) phases were extracted and analyzed separately. Volume was reduced to approximately $10 \mathrm{~mL}$ using a rotoevaporator, followed by evaporation under high purity nitrogen to a final volume of $200 \mu \mathrm{L}$.

Water, sediment and air filter extracts were analyzed for PFSAs, PFCAs and FTUCAs via liquid chromatography - tandem mass spectrometry (LC-MS/MS). Both water and sediment samples were quantified using matrix-matched calibration standards (in the case of sediment, these were created using sediment from the bottom $5 \mathrm{~cm}$ of the sediment core) and isotopicallylabeled standards. Final sediment concentrations were reported as dry weight values.

Air sample extracts were analyzed for FTOHs and FSAs using gas chromatography mass spectrometry (GC-MS). Following analysis of volatile analytes on the GC-MS, the air filter extracts were transferred, using approximately $10 \mathrm{~mL}$ methanol, to $15 \mathrm{~mL}$ polypropylene Falcon tubes (VWR, Mississauga), blown to dryness under a gentle stream of high purity nitrogen and reconstituted with $0.5 \mathrm{~mL}$ of methanol. These solvent exchanged air filter extracts were then analyzed for non-volatile analytes via LC-MS/MS as described above.

Blank samples, consisting of methanol, extraction of sediment from the bottom $5 \mathrm{~cm}$ of the sediment cores, or extraction of clean air sampling media were conducted with each set of samples extracted and analyzed. In both LC-MS/MS and GC-MS analyses, standards and solvent blanks were included every 5 samples during analysis to monitor for changes in instrument sensitivity and possible contamination.

As discussed in the manuscript, the extraction method used for the air samples was designed for volatile precursor analytes (FSAs and FTOHs), and analysis of non-volatile analytes was a secondary consideration. Extraction recovery of non-volatile analytes spiked onto clean 
filters $(n=4)$ was generally acceptable, but was lower than desired for some analytes, ranging from $27 \%$ (PFHpA) to $103 \%(10: 2$ FTUCA)(Table S2). In addition, isotopically-labeled PFOS, PFOA, PFNA and perfluorodecanoate (PFDA) spiked onto filters $(n=3)$ prior to sampling an average of $675 \mathrm{~m}^{3}$ of air, were also recovered. Recoveries of isotopically-labeled PFOS and PFOA, were acceptable ranging from 110 and $73 \%$ respectively, while recoveries of isotopicallylabeled PFNA and PFDA were less, 55 and 53 \% respectively, likely due to the increased chain length of the molecules. As such, we report values for PFSAs and PFCAs on filters, but caution that reported concentrations may be under represented. 
Table S1. Characteristics of Amituk, Char and Resolute Lakes.

\begin{tabular}{cccc} 
& Amituk Lake & Char Lake & Resolute Lake \\
\cline { 2 - 4 } Latitude & $75^{\circ} 03^{\prime} \mathrm{N}$ & $74^{\circ} 42^{\prime} \mathrm{N}$ & $74^{\circ} 41^{\prime} \mathrm{N}$ \\
Longitude & $93^{\mathrm{o}} 49^{\prime} \mathrm{W}$ & $94^{\mathrm{o}} 53^{\prime} \mathrm{W}$ & $94^{\mathrm{o}} 55^{\prime} \mathrm{W}$ \\
Lake Area $\left(\mathrm{km}^{2}\right)$ & $0.378^{\mathrm{a}}$ & $0.526^{\mathrm{a}}$ & $1.27^{\mathrm{a}}$ \\
Watershed Area (km $\left.{ }^{2}\right)$ & $26.6^{\mathrm{a}}$ & $4.9^{\mathrm{a}}$ & $23^{\mathrm{a}}$ \\
Maximum Depth (m) & $42^{\mathrm{b}}$ & $27.5^{\mathrm{c}}$ & $15.6^{\mathrm{c}}$ \\
Mean Depth (m) & $19.4^{\mathrm{d}}$ & $10.2^{\mathrm{d}}$ & $\sim 7^{\mathrm{c}}$ \\
Residence time (years) & $1.4^{\mathrm{e}}$ & $9.0-14^{\mathrm{e}}$ & unknown
\end{tabular}

${ }^{\mathrm{a}}$ Muir et al, 2005 (10)

${ }^{\mathrm{b}}$ Muir et al, 1995 (11)

${ }^{\mathrm{c}}$ Markager et al, 1999 (and references within) (12)

${ }^{\mathrm{d}}$ Freitas et al, 1997 (and references within) (13)

${ }^{\text {e }}$ Law et al, 2001 (and references within) (14) 
Table S2. Limits of detection (LODs), limits of quantitation (LOQ) and extraction recoveries for analytes of interest in water, sediment and air samples. LODs were defined as three times the standard deviation of the average blank response. LOQs were defined as ten time the average standard deviation of the blank response or the lowest standard on the calibration curve.

\begin{tabular}{|c|c|c|c|c|c|c|c|c|c|c|c|c|c|c|}
\hline & \multirow{3}{*}{$\begin{array}{c}\text { Water } \\
\text { LOQ (ng/L) }\end{array}$} & \multicolumn{4}{|c|}{ Sediment } & \multicolumn{9}{|c|}{ Air } \\
\hline & & \multirow[t]{2}{*}{$\%$ recovery } & \multicolumn{3}{|c|}{ LOD (ng/g dry weight) } & \multicolumn{3}{|c|}{ LOQ (ng/g dry weight) } & \multirow{2}{*}{$\%$ recovery } & \multicolumn{2}{|c|}{$\operatorname{LOD}\left(\mathrm{pg} / \mathrm{m}^{3}\right)$} & \multicolumn{2}{|c|}{ LOQ $\left(\mathrm{pg} / \mathrm{m}^{3}\right)$} & \multirow[t]{2}{*}{$\%$ recovery } \\
\hline & & & Amituk Lake & Char Lake & Resolute Lake & Amituk Lake & Char Lake & Resolute Lake & & Filter* & PUF-XAD* & Filter* & PUF-XAD* & \\
\hline PFBS & n.d. & n.d. & 0.029 & 1.0 & 0.046 & 0.059 & 1.1 & 0.092 & 82 & n.d. & $\mathrm{n} / \mathrm{a}$ & n.d. & $\mathrm{n} / \mathrm{a}$ & n.d. \\
\hline PFHS & 1.4 & 94 & 0.058 & 0.54 & 0.046 & 0.12 & 0.55 & 0.046 & 77 & 0.44 & $\mathrm{n} / \mathrm{a}$ & 0.13 & $\mathrm{n} / \mathrm{a}$ & 61 \\
\hline PFOS & 0.5 & 92 & 0.31 & 0.29 & 0.29 & 0.59 & 0.35 & 0.46 & 72 & 0.88 & $\mathrm{n} / \mathrm{a}$ & 0.27 & $\mathrm{n} / \mathrm{a}$ & 77 \\
\hline PFDS & 0.5 & 102 & 0.029 & 0.25 & 0.86 & 0.059 & 0.53 & 0.92 & 62 & 0.34 & $\mathrm{n} / \mathrm{a}$ & 0.10 & $\mathrm{n} / \mathrm{a}$ & 50 \\
\hline PFHA & 1.6 & 95 & 0.15 & 2.6 & 0.18 & 0.29 & 3.3 & 0.18 & 138 & 1.3 & $\mathrm{n} / \mathrm{a}$ & 1.9 & $\mathrm{n} / \mathrm{a}$ & 27 \\
\hline PFOA & 1.3 & 106 & 0.16 & 1.1 & 1.7 & 0.29 & 1.1 & 1.8 & 124 & 0.87 & $\mathrm{n} / \mathrm{a}$ & 1.9 & $\mathrm{n} / \mathrm{a}$ & 75 \\
\hline PFNA & 1.2 & 108 & 0.048 & 0.47 & 2.5 & 0.059 & 0.55 & 3.1 & 78 & 0.23 & $\mathrm{n} / \mathrm{a}$ & 0.37 & $\mathrm{n} / \mathrm{a}$ & 82 \\
\hline PFDA & 0.8 & 93 & 0.024 & 0.26 & 0.43 & 0.059 & 0.55 & 0.46 & 116 & 0.69 & $\mathrm{n} / \mathrm{a}$ & 0.74 & $\mathrm{n} / \mathrm{a}$ & 41 \\
\hline PFUA & 1.4 & 99 & 0.020 & 0.055 & 0.21 & 0.059 & 0.11 & 0.46 & 90 & 0.09 & $\mathrm{n} / \mathrm{a}$ & 0.31 & $\mathrm{n} / \mathrm{a}$ & 51 \\
\hline PFDoA & 0.9 & 107 & 0.12 & 0.40 & 0.34 & 0.29 & 0.55 & 0.46 & 79 & 0.33 & $\mathrm{n} / \mathrm{a}$ & 0.74 & $\mathrm{n} / \mathrm{a}$ & 60 \\
\hline PFTriA & 2.0 & n.d. & 0.029 & 0.055 & 0.046 & 0.059 & 0.11 & 0.092 & n.d. & 0.04 & $\mathrm{n} / \mathrm{a}$ & 0.14 & $\mathrm{n} / \mathrm{a}$ & n.d. \\
\hline PFTetA & 2.0 & n.d. & 0.049 & 0.56 & 0.099 & 0.059 & 1.1 & 0.18 & 72 & 0.21 & $\mathrm{n} / \mathrm{a}$ & 0.37 & $\mathrm{n} / \mathrm{a}$ & 22 \\
\hline PFPA & 2.0 & n.d. & 0.029 & 0.055 & 0.046 & 0.059 & 0.11 & 0.092 & n.d. & 0.04 & $\mathrm{n} / \mathrm{a}$ & 0.37 & $\mathrm{n} / \mathrm{a}$ & n.d. \\
\hline 8:2 FTUCA & 2.0 & 95 & 0.030 & 0.19 & 0.32 & 0.59 & 0.22 & 0.92 & 66 & 0.08 & $\mathrm{n} / \mathrm{a}$ & 0.26 & $\mathrm{n} / \mathrm{a}$ & 74 \\
\hline 10:2 FTUCA & 2.0 & 105 & 0.029 & 0.55 & 0.46 & 0.059 & 0.55 & 0.46 & 66 & 0.08 & $\mathrm{n} / \mathrm{a}$ & 0.27 & $\mathrm{n} / \mathrm{a}$ & 103 \\
\hline 6:2 FTOH & $\mathrm{n} / \mathrm{a}$ & $\mathrm{n} / \mathrm{a}$ & $\mathrm{n} / \mathrm{a}$ & $\mathrm{n} / \mathrm{a}$ & $\mathrm{n} / \mathrm{a}$ & $\mathrm{n} / \mathrm{a}$ & $\mathrm{n} / \mathrm{a}$ & $\mathrm{n} / \mathrm{a}$ & $\mathrm{n} / \mathrm{a}$ & 6.3 & 5.4 & 11.3 & 13.6 & 82 \\
\hline 8:2 FTOH & $\mathrm{n} / \mathrm{a}$ & $\mathrm{n} / \mathrm{a}$ & $\mathrm{n} / \mathrm{a}$ & $\mathrm{n} / \mathrm{a}$ & $\mathrm{n} / \mathrm{a}$ & $\mathrm{n} / \mathrm{a}$ & $\mathrm{n} / \mathrm{a}$ & $\mathrm{n} / \mathrm{a}$ & $\mathrm{n} / \mathrm{a}$ & 3.5 & 4.3 & 5.0 & 2.3 & 86 \\
\hline 10:2 FTOH & $\mathrm{n} / \mathrm{a}$ & $\mathrm{n} / \mathrm{a}$ & $\mathrm{n} / \mathrm{a}$ & $\mathrm{n} / \mathrm{a}$ & $\mathrm{n} / \mathrm{a}$ & $\mathrm{n} / \mathrm{a}$ & $\mathrm{n} / \mathrm{a}$ & $\mathrm{n} / \mathrm{a}$ & $\mathrm{n} / \mathrm{a}$ & 2.2 & 1.0 & 3.0 & 1.5 & 86 \\
\hline NMeFBSE & n/a & n/a & $\mathrm{n} / \mathrm{a}$ & $\mathrm{n} / \mathrm{a}$ & $\mathrm{n} / \mathrm{a}$ & $\mathrm{n} / \mathrm{a}$ & $\mathrm{n} / \mathrm{a}$ & $\mathrm{n} / \mathrm{a}$ & $\mathrm{n} / \mathrm{a}$ & 0.7 & 17.7 & 1.5 & 24.9 & 119 \\
\hline NEtFBSE & $\mathrm{n} / \mathrm{a}$ & n/a & $\mathrm{n} / \mathrm{a}$ & $\mathrm{n} / \mathrm{a}$ & $\mathrm{n} / \mathrm{a}$ & $\mathrm{n} / \mathrm{a}$ & n/a & $\mathrm{n} / \mathrm{a}$ & $\mathrm{n} / \mathrm{a}$ & 4.9 & 15.5 & 18.9 & 18.5 & 117 \\
\hline PFOSA & $n / a$ & n/a & $n / a$ & $\mathrm{n} / \mathrm{a}$ & $n / a$ & $\mathrm{n} / \mathrm{a}$ & $n / a$ & $\mathrm{n} / \mathrm{a}$ & $\mathrm{n} / \mathrm{a}$ & 1.5 & 6.7 & 1.2 & 8.0 & 96 \\
\hline NEtFOSA & $n / a$ & $n / a$ & $\mathrm{n} / \mathrm{a}$ & $\mathrm{n} / \mathrm{a}$ & $n / a$ & $n / a$ & n/a & $\mathrm{n} / \mathrm{a}$ & $\mathrm{n} / \mathrm{a}$ & 3.9 & 6.9 & 5.6 & 9.3 & 109 \\
\hline NMeFOSE & $\mathrm{n} / \mathrm{a}$ & $\mathrm{n} / \mathrm{a}$ & $\mathrm{n} / \mathrm{a}$ & $\mathrm{n} / \mathrm{a}$ & $\mathrm{n} / \mathrm{a}$ & $\mathrm{n} / \mathrm{a}$ & $\mathrm{n} / \mathrm{a}$ & $\mathrm{n} / \mathrm{a}$ & $\mathrm{n} / \mathrm{a}$ & 6.2 & 20.8 & 9.7 & 29.4 & 120 \\
\hline NEtFOSE & $\mathrm{n} / \mathrm{a}$ & $\mathrm{n} / \mathrm{a}$ & $\mathrm{n} / \mathrm{a}$ & $\mathrm{n} / \mathrm{a}$ & $\mathrm{n} / \mathrm{a}$ & $\mathrm{n} / \mathrm{a}$ & $\mathrm{n} / \mathrm{a}$ & $\mathrm{n} / \mathrm{a}$ & $\mathrm{n} / \mathrm{a}$ & 9.4 & 20.4 & 13.2 & 26.6 & 122 \\
\hline
\end{tabular}


Table S3. Concentrations of PFSAs and PFCAS in water samples.

\begin{tabular}{|c|c|c|c|c|c|c|c|c|c|c|c|c|c|}
\hline Location & Sampling Date & & PFHxS & PFOS & PFDS & PFHрA & PFOA & PFNA & PFDA & PFUA & PFDoA & 8:2 FTUCA & 10:2 FTUCA \\
\hline \multirow[t]{3}{*}{ Amituk Lake } & August 2003 & Sample 1 & nd & 0.9 & nd & 0.8 & 2.0 & 0.4 & 1.4 & 3.4 & 0.6 & 2.4 & nd \\
\hline & & Sample 2 & nd & 1.5 & nd & nd & 1.9 & 0.2 & 1.4 & 2.6 & 0.5 & 1.8 & nd \\
\hline & & Sample 3 & nd & 1.2 & nd & 1.0 & 8.4 & 0.2 & 0.5 & 1.4 & nd & nd & 3.2 \\
\hline \multirow[t]{6}{*}{ Char Lake } & August 2003 & Sample 1 & nd & 1.1 & nd & 0.3 & 3.4 & 1.4 & 5.4 & 4.1 & nd & 5.8 & nd \\
\hline & & Sample 2 & nd & 2.3 & nd & 0.5 & 2.5 & nd & nd & 5.9 & nd & nd & nd \\
\hline & & Sample 3 & nd & 2.1 & nd & nd & 1.8 & nd & 7.3 & 4.7 & nd & nd & nd \\
\hline & July 2005 & Sample 1 & nd & 0.9 & nd & nd & 0.4 & 2.5 & 10 & 2.7 & nd & nd & 9.2 \\
\hline & & Sample 2 & nd & 2.1 & nd & 1.6 & 1.9 & 1.8 & 13 & 3.8 & nd & nd & nd \\
\hline & & Sample 3 & nd & 2.5 & nd & nd & 0.5 & 0.2 & 7.5 & 3.9 & nd & nd & nd \\
\hline \multirow[t]{12}{*}{ Resolute Lake } & August 2003 & Sample 1 & 19 & 49 & 1.0 & 49 & 13 & 6.1 & 2.4 & 1.0 & 2.3 & nd & nd \\
\hline & & Sample 2 & 24 & 90 & nd & 25 & 16 & nd & nd & 2.7 & nd & nd & nd \\
\hline & & Sample 3 & 19 & 68 & nd & 18 & 12 & nd & 2.9 & 5.0 & nd & nd & nd \\
\hline & July 2005 & Sample 1 & 1.5 & 23 & nd & 3.1 & 6.0 & 2.3 & 8.7 & 4.5 & 0.5 & nd & 7.9 \\
\hline & & Sample 2 & 5.4 & 23 & nd & 7.0 & 5.8 & 1.5 & 5.6 & 4.0 & 0.7 & nd & 11 \\
\hline & & Sample 3 & 1.6 & 23 & nd & 6.9 & 5.0 & 1.2 & 4.6 & 3.6 & nd & 4.0 & nd \\
\hline & August 12005 & Sample 1 & 10 & 43 & nd & 14 & 9.9 & 3.0 & 14 & 4.3 & 0.9 & nd & nd \\
\hline & & Sample 2 & 13 & 43 & nd & 14 & 11 & 2.8 & 20 & 4.5 & nd & nd & nd \\
\hline & & Sample 3 & 17 & 41 & nd & 9.3 & 9.5 & 2.5 & 3.3 & 4.8 & nd & 15.1 & nd \\
\hline & August 82005 & Sample 1 & 14 & 44 & nd & 6.2 & 9.0 & 2.9 & 29 & 5.8 & nd & nd & nd \\
\hline & & Sample 2 & 13 & 46 & nd & 9.4 & 11 & 1.3 & 8.7 & 5.5 & nd & nd & nd \\
\hline & & Sample 3 & 15 & 46 & nd & 15 & 10 & 0.9 & 14 & 3.5 & nd & nd & nd \\
\hline \multirow[t]{3}{*}{ Meretta Lake } & July 2005 & Sample 1 & 17 & 55 & 11 & 29 & 13 & 4.4 & 24 & 0.5 & 0.5 & 3.8 & nd \\
\hline & & Sample 2 & 18 & 57 & 11 & 22 & 15 & 3.8 & 18 & 0.2 & nd & nd & nd \\
\hline & & Sample 3 & 12 & 56 & 10 & 26 & 14 & 4.3 & 16 & 0.2 & nd & nd & nd \\
\hline \multirow{3}{*}{$\begin{array}{l}\text { Outflow from } \\
\text { Meretta Lake }\end{array}$} & July 2005 & Sample 1 & 12 & 50 & 13 & 22 & 13 & 3.3 & 7.2 & 0.5 & nd & nd & nd \\
\hline & & Sample 2 & 8.9 & 53 & 14 & 17 & 13 & 4.2 & 14 & 0.4 & nd & nd & nd \\
\hline & & Sample 3 & 12 & 53 & 14 & 21 & 13 & 3.0 & 8.7 & 0.2 & nd & 4.2 & nd \\
\hline
\end{tabular}


Table S4. Concentrations of PFSAs and PFCAs in sediment samples.

\begin{tabular}{|c|c|c|c|c|c|c|c|c|c|c|c|c|c|c|c|}
\hline & PFBS & PFHxS & PFOS & PFDS & РFНрА & PFOA & PFNA & PFDA & PFUA & PFDoA & PFTriA & PFTetA & PFPA & 8:2 FTUCA & 10:2 FTUCA \\
\hline \multicolumn{16}{|l|}{ Resolute Lake } \\
\hline $0-1 \mathrm{~cm}$ & nd & 3.5 & 85 & nd & 6.8 & 7.5 & 3.2 & $<0.46$ & $<0.46$ & nd & nd & nd & nd & nd & nd \\
\hline $1-2 \mathrm{~cm}$ & 0.11 & 1.3 & 33 & nd & 0.95 & 2.3 & $<3.1$ & $<0.46$ & $<0.46$ & nd & nd & nd & nd & $<0.92$ & nd \\
\hline $2-3 \mathrm{~cm}$ & nd & 1.2 & 24 & nd & $<0.18$ & $<1.8$ & $<2.55$ & $<0.46$ & nd & $<0.46$ & nd & nd & nd & $<0.92$ & nd \\
\hline \multicolumn{16}{|l|}{ Char Lake } \\
\hline $0-1 \mathrm{~cm}$ & $<1.1$ & nd & 1.1 & $<0.53$ & $<3.3$ & 1.7 & $<0.55$ & 0.60 & nd & $<0.55$ & nd & nd & nd & $<0.22$ & 1.9 \\
\hline $1-2 \mathrm{~cm}$ & nd & $<0.55$ & $<0.35$ & $<0.53$ & $<3.3$ & $<1.1$ & nd & $<0.55$ & nd & $<0.55$ & nd & nd & nd & $<0.22$ & nd \\
\hline $2-3 \mathrm{~cm}$ & $<1.1$ & nd & $<0.35$ & $<0.53$ & $<3.3$ & 1.2 & $<0.55$ & $<0.55$ & nd & $<0.55$ & nd & nd & nd & nd & nd \\
\hline \multicolumn{16}{|l|}{ Amituk Lake } \\
\hline $0-1.5 \mathrm{~cm}$ & 0.047 & 1.0 & 0.062 & nd & 3.9 & 0.96 & 0.35 & 0.12 & 0.056 & $<0.29$ & nd & $<0.059$ & nd & $<0.59$ & 0.46 \\
\hline $1.5-2.5 \mathrm{~cm}$ & 0.068 & 0.70 & 0.027 & nd & 3.0 & 0.63 & 0.23 & 0.071 & 0.072 & $<0.29$ & nd & $<0.059$ & nd & $<0.59$ & 0.18 \\
\hline $2.5-3.5 \mathrm{~cm}$ & nd & nd & 0.022 & nd & $<2.9$ & $<0.29$ & $<0.059$ & $<0.059$ & $<0.059$ & $<0.29$ & nd & $<0.059$ & nd & $<0.59$ & nd \\
\hline
\end{tabular}


Table S5. Concentrations $\left(\mathrm{pg} / \mathrm{m}^{3}\right)$ of individual FTOHs and FSAs observed in both particle (filter) and gas (PUF-XAD) phases. Values in brackets were $<$ LOQ, but $>$ LOD.

$\begin{array}{llllllll}\text { sample volume }\left(\mathrm{m}^{3}\right) & \text { 6:2 FTOH } & \text { 8:2FTOH } & \text { 10:2 FTOH } & \text { NMEFBSE } & \text { NEtFBSE } & \text { NEtFOSA NMeFOSE NEtFOSE } & \text { PFOSA }\end{array}$

\section{Filter Samples}

$\begin{array}{ccccc}\text { July } 4 & 337 & <11.3 & <5.2 & 4.1 \\ \text { July 7 } & 437 & <11.3 & <5.2 & <3.0 \\ \text { July 10 } & 465 & <11.3 & <5.2 & <3.0 \\ \text { July 13 } & 510 & <11.3 & <5.2 & <3.0 \\ \text { July 18 } & 474 & <11.3 & <5.2 & <3.0 \\ \text { July 21 } & 412 & <11.3 & <5.2 & <3.0 \\ \text { July 24 } & 418 & <11.3 & <5.2 & <3.0 \\ \text { July 27 } & 1768 & <11.3 & <5.2 & <3.0 \\ \text { Aug 4 } & 1113 & <11.3 & <5.2 & <3.0 \\ \text { Aug 8 } & 817 & & & \end{array}$

PUF-XAD Samples

$\begin{array}{lr}\text { July } 4 & 337 \\ \text { July } 7 & 437 \\ \text { July } 10 & 465 \\ \text { July } 13 & 510 \\ \text { July } 18 & 474 \\ \text { July } 21 & 412 \\ \text { July } 24 & 418 \\ \text { July } 27 & 1768 \\ \text { Aug } 4 & 1113 \\ \text { Aug } 8 & 817\end{array}$

$\begin{array}{ccc}<13.6 & <2.3 & <1.5 \\ <13.6 & <2.3 & <1.5 \\ <13.6 & <2.3 & <1.5 \\ (8.0) & <2.3 & <1.5 \\ <13.6 & <2.3 & <1.5 \\ (6.5) & 16.6 & <1.5 \\ <13.6 & 18.6 & <1.5 \\ 19.7 & 15.1 & <1.5 \\ <13.6 & 18.4 & <1.5 \\ <13.6 & 10.9 & 3.5\end{array}$

$\begin{array}{cc}5.4 & (17.9) \\ \text { nd } & \text { nd } \\ \text { nd } & \text { nd } \\ \text { nd } & (6.6) \\ \text { nd } & <18.9 \\ 4.4 & <18.9 \\ \text { nd } & \text { nd } \\ \text { nd } & <18.9 \\ 2.2 & <18.9\end{array}$

$\begin{array}{lccc}(4.4) & 18.0 & 17.7 & 6.2 \\ (5.3) & 26.9 & 27.9 & 4.7 \\ <5.6 & <9.7 & <13.2 & <1.5 \\ <5.6 & (6.4) & <13.2 & <1.5 \\ <5.6 & <9.7 & <13.2 & 1.6 \\ <5.6 & <9.7 & <13.2 & 3.0 \\ <5.6 & <9.7 & <13.2 & 2.7 \\ <5.6 & <9.7 & <13.2 & <1.5 \\ <5.6 & <9.7 & <13.2 & <1.5 \\ <5.6 & <9.7 & <13.2 & <1.5\end{array}$

$<18.9$

nd
$<24.9$
$<24.9$
nd
$<24.9$
$<24.9$
$<24.9$
34.0
$<24.9$
$(22.7)$

$<18.5$
$<18.5$
$<18.5$
$<18.5$
$<18.5$
$<18.5$
$<18.5$
$<18.5$
$<18.5$
$<18.5$

$<29.4$
$<29.4$
$<29.4$
$<29.4$
$<29.4$
34.3
$(31.2)$
39.2
$<29.4$
$(27.2)$

$\begin{array}{ll}<26.6 & <8.0 \\ <26.6 & <8.0 \\ <26.6 & <8.0 \\ <26.6 & <8.0 \\ <26.6 & <8.0 \\ <26.6 & 61.3 \\ <26.6 & 42.1 \\ <26.6 & 30.0 \\ <26.6 & 14.1 \\ <26.6 & 9.7\end{array}$




\section{Back Trajectory Calculations}

Three-day back trajectories were calculated for the air sampling site at $10 \mathrm{~m}$ above ground level at 6 hour intervals for each day air samples were collected, using the Canadian Meteorological Centre (CMC) Trajectory Model. This information was used to produce a back trajectory probability map or air shed (15), and is useful in identifying those regions where the parcels of air arriving at the sampling site were most frequently associated with.

Figure S1. Back trajectory probability map/air shed of air sampling location near Resolute Bay on Cornwallis Island, Nunavut, Canada. Samples collected July and August 2004.

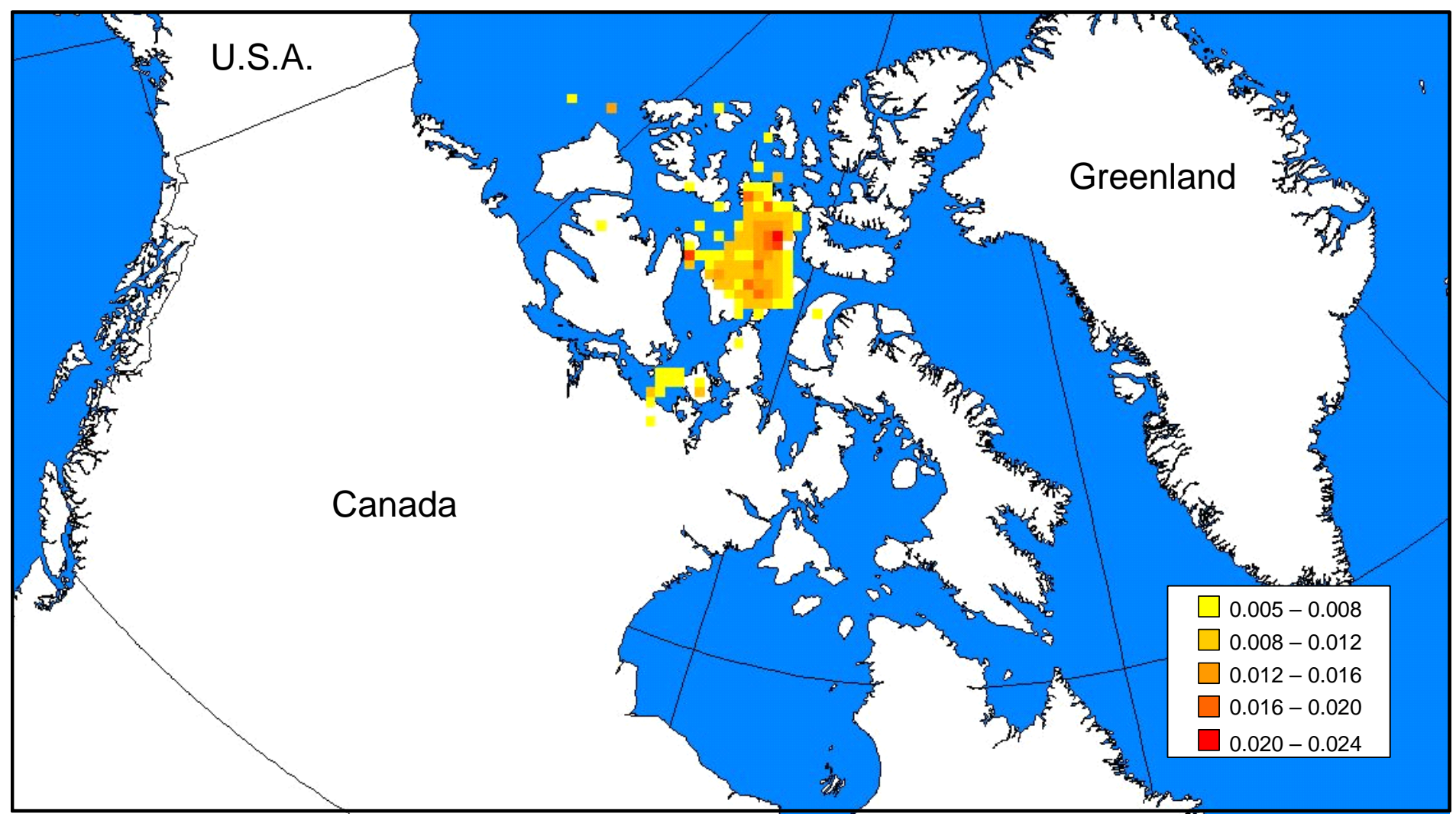


Table S6. Concentrations of chlorophyll A ( $\mu \mathrm{g} / \mathrm{L})$, chloride (mg/L) and sodium (mg/L) in Lakes of Cornwallis Island, Nunavut, Canada. Adapted from Muir and Wang, 2005 (16)

Chlorophyll A Chloride Sodium

\begin{tabular}{ccccc} 
Location & Sampling Date & ug/L & $\mathrm{mg} / \mathrm{L}$ & $\mathrm{mg} / \mathrm{L}$ \\
\hline & & & & \\
Small Lake & July 25 2005 & $<0.1$ & 11.2 & 5.71 \\
North Lake & July 25 2005 & $<0.1$ & 1.98 & 0.99 \\
Char Lake & July 22 2005 & 0.2 & 10.4 & 7.11 \\
Resolute Lake & July 22 2005 & 0.2 & 19.6 & 11.4 \\
Meretta Lake & July 26 2005 & $<0.1$ & 7.9 & 4.07 \\
Aqiatusuk Lake & July 22 2005 & $<0.1$ & 2.26 & 1.13 \\
12 Mile Lake & July 23 2005 & 0.3 & 5.53 & 2.71 \\
Amituk Lake & July 26 2005 & $<0.1$ & 1.95 & 0.92
\end{tabular}




\section{LITERATURE CITED}

(1) Bidleman, T. F.; Falconer, R. L.; Walla, M. D. 1995. Toxaphene and other organochlorine compounds in air and water at Resolute Bay, N.W.T. Sci. Tot. Environ. 160/161, 55-63.

(2) Falconer, R. L.; Bidleman, T. F.; Gregor, D. J. 1995. Air-water gas exchange and evidence for metabolism of hexachlorocyclohexanes in Resolute Bay, NWT. Sci. Tot. Environ. 160/161, 65-74.

(3) Stock, N. L.; Lau, F. K.; Ellis, D. A.; Martin, J. W.; Muir, D. G. C.; Mabury, S. A. Polyfluorinated telomer alcohols and sulfonamides in the North American troposphere. Environ. Sci. Technol. 2004, 991-996.

(4) Yang, F. ${ }^{210} \mathrm{~Pb}$ Dating of Lacustrine Sediments from Arctic Lakes (Core 293), Nunavut. Environment Canada: Burlington, ON, 2005.

(5) Appleby, P. G.; Oldfield, F. The calculation of ${ }^{210} \mathrm{~Pb}$ dates assuming a constant supply of unsupported ${ }^{210} \mathrm{~Pb}$ to the sediment. Catena 1978, 5, 1-8.

(6) Higgins, C. P.; Luthy, R.G. Sorption of perfluorinated surfactants on sediments. Environ. Sci. Technol. 2006, 40, 7251-7256.

(7) Stock, N. L.; Mabury, S. A.; Muir, D. C. G. Perflluoroalkyl contaminants in Lake Ontario sediment: contribution to mass balance. Environ. Sci. Technol. 2007, In preparation.

(8) Furdui, V. I.; Crozier, P. W.; Reiner, E. J.; Mabury, S. A. Optimized trace level analysis of perfluorinated acids in Great Lakes watershed. Organhalo. Comp. 2005, 67, 211-214.

(9) Martin, J. W.; Muir, D. G. C.; Moody, C. A.; Ellis, D. A.; Kwan, W. C.; Solomon, K. R.; Mabury, S. A. Collection of airborne fluorinated organics and analysis by gas chromatography/chemical ionization mass spectrometry. Anal. Chem. 2002, 74, 584-590.

(10) Muir, D. C. G.; Wang, X.; Bright, D.; Lockhart, W. L.; Koch, G. Spatial and temporal trends of mercury and other metals in landlocked char from lakes in the Canadian Arctic archipelago. Sci. Tot. Environ. 2005, 351-352, 464-478.

(11) Muir, D. C. G.; Grift, N. P.; Lockhart, W. L.; Wilkinson, P.; Billeck, B. N.; Brunskill, G. J. Spatial trends and historical profiles of organochlorine pesticides in Arctic lake sediments. Sci. Tot. Environ. 1995, 160-161, 447-457.

(12) Markager, S.; Vincent, W. F.; Tang, E. P. Y. Carbon fixation by phytoplankton in high Arctic lakes: Implications of low temperature for photosynthesis. Limnol. Oceanogr. 1999, 44, 597-607.

(13) Freitas, H.; Diamond, M.; Semkin, R.; Gregor, D. Contaminant fate in high arctic lakes: development and application of a mass balance model. Sci. Tot. Environ. 1997, 201, 171187. 
(14) Law, S. A.; Diamond, M. L.; Helm, P. A.; Jantunen, L. M.; Alaee, M. Factors affecting the occurrence and enantiomeric degradation of hexachlorocyclohexane isomers in northern and temperate aquatic systems. Environ. Tox. Chem. 2001, 20, 2690-2698.

(15) Gouin, T.; Harner, T.; Blanchard, P.; Mackay, D. Passive and active air samplers as complementary methods for investigating persistent organic pollutants in the Great Lakes Basin. Environ. Sci. Technol. 2005, 39, 9115-9122.

(16) Muir D. C. G.; Wang, X. Water Chemistry of Arctic Lakes. Environment Canada: Burlington, ON, 2005 